\title{
Mid-IR heterogeneous silicon photonics
}

\author{
Gunther Roelkens, ${ }^{1,}$ Utsav Dave ${ }^{1}$, Alban Gassenq, ${ }^{1}$ Nannicha Hattasan, ${ }^{1}$ Chen Hu, ${ }^{1}$ Bart Kuyken, ${ }^{1}$ Francois Leo, ${ }^{1}$ Aditya \\ Malik, ${ }^{1}$ Muhammad Muneeb, ${ }^{1}$ Eva Ryckeboer, ${ }^{1}$ Sarah Uvin ${ }^{1}$, Zeger Hens, ${ }^{1}$ Roel Baets,${ }^{1}$ Yosuke Shimura, ${ }^{2}$ Federica \\ Gencarelli, ${ }^{2}$ Benjamin Vincent, ${ }^{2}$ Roger Loo, ${ }^{2}$ Joris Van Campenhout, ${ }^{2}$ Laurent Cerutti, ${ }^{3}$ Jean-Baptiste Rodriguez, ${ }^{3}$ Eric \\ Tournié, ${ }^{3}$ Xia Chen, ${ }^{4}$ Milos Nedeljkovic, ${ }^{4}$ Goran Mashanovich, ${ }^{4}$ Li Shen, ${ }^{4}$ Noel Healy, ${ }^{4}$ Anna C. Peacock,${ }^{4}$ Xiaoping \\ Liu, ${ }^{5}$ Richard Osgood, ${ }^{5}$ and William Green ${ }^{6}$ \\ ${ }^{1}$ Photonics Research Group - Center for nano- and biophotonics (NB-Photonics), Sint-Pietersnieuwstraat 41, B-9000 Ghent, Belgium \\ ${ }^{2}$ imec, Kapeldreef 75, B-3001 Leuven, Belgium \\ ${ }^{3}$ Institut d'Electronique, UMR 5214, Université Montpellier 2 - CNRS, Montpellier, France \\ ${ }^{4}$ Optoelectronics Research Centre, Faculty of Physical Sciences and Engineering, University of Southampton, Southampton, SO17 1BJ United \\ Kingdom \\ ${ }^{5}$ Department of Electrical Engineering, Columbia University, New York 10027, USA \\ ${ }^{6}$ IBM T.J. Watson Research Center, 1101 Kitchawan Road, Yorktown Heights, New York 10598, USA
}

"gunther.roelkens@intec.ugent.be

\begin{abstract}
In this paper we discuss silicon-based photonic integrated circuit technology for applications beyond the telecommunication wavelength range. Silicon-on-insulator and germanium-on-silicon passive waveguide circuits are described, as well as the integration of III-V semiconductors, IV-VI colloidal nanoparticle films and GeSn alloys on these circuits for increasing the functionality. The strong nonlinearity of silicon combined with the low nonlinear absorption in the mid-infrared is exploited to generate picosecond pulse based supercontinuum sources and optical parametric oscillators that can be used as spectroscopic sensor sources.
\end{abstract}

Keywords: mid-infrared spectroscopy; photonic integration

\section{INTRODUCTION}

Silicon photonics is emerging as a compelling platform for optical interconnect and telecommunication applications, mainly driven by the scalability of the CMOS manufacturing technology, resulting in low-cost, high-volume and fairly complex photonic integrated circuits [1]. Besides using silicon photonics for optical communication, there is also a lot of interest in implementing sensing functions on a silicon chip. Nowadays, these systems rely on the chemical functionalization of silicon resonators, which upon binding with the molecule of interest, results in a resonance wavelength shift that can be detected [2]. While the sensitivity of such a sensor is mostly determined by the optical design of the cavity, the selectivity of the sensor is completely determined by the functionalization chemistry, which tends to degrade over time and needs to be redeveloped for every molecule of interest. Therefore, sensors that detect intrinsic properties of molecules, such as their fingerprint absorption spectrum, integrated on a silicon photonic circuit would allow for a new class of highly selective and sensitive devices. Since absorption spectra of small molecules are strongest in the mid-infrared wavelength range, mid-infrared silicon photonic circuit technology could make an important impact in this field [3]. In this paper we will describe our work in the field of heterogeneous silicon photonic integrated circuits for the mid-infrared. Silicon-on-insulator and germanium-on-silicon passive waveguide circuits are described, as well as the integration of III-V semiconductors, IV-VI colloidal nanoparticle films and GeSn alloys on these circuits for increasing the functionality. The strong nonlinearity of silicon combined with the low nonlinear absorption in the mid-infrared is exploited to generate picosecond pulse based supercontinuum sources and optical parametric oscillators that can be used as spectroscopic sensor sources.

\section{MID-IR PASSIVE WAVEGUIDE TECHNOLOGY}

There are many approaches to realize mid-infrared photonic integrated circuits based on silicon technology. The most straightforward approach is to use silicon-on-insulator waveguide structures, since this is also the platform for telecommunication applications. Without changing anything to the platform we were able to demonstrate silicon-oninsulator low loss waveguides $(0.5 \mathrm{~dB} / \mathrm{cm}$ in the $2-2.5 \mu \mathrm{m}$ wavelength range) [4] and spectrometers operating in the 
short-wave infrared $(2-2.5 \mu \mathrm{m})$ and the mid-infrared (around $3.8 \mu \mathrm{m})$ [5]. Spectrometers based on echelle gratings and arrayed waveguide gratings were demonstrated, as shown in Figure 1.

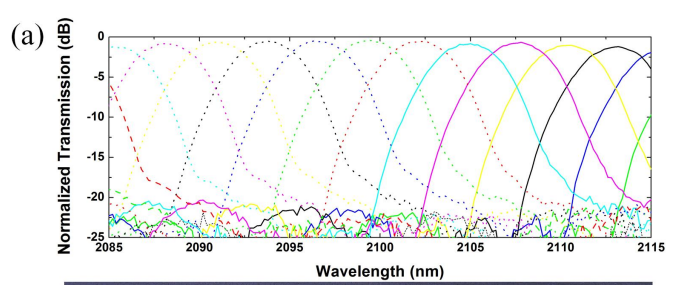

(b) 뜽
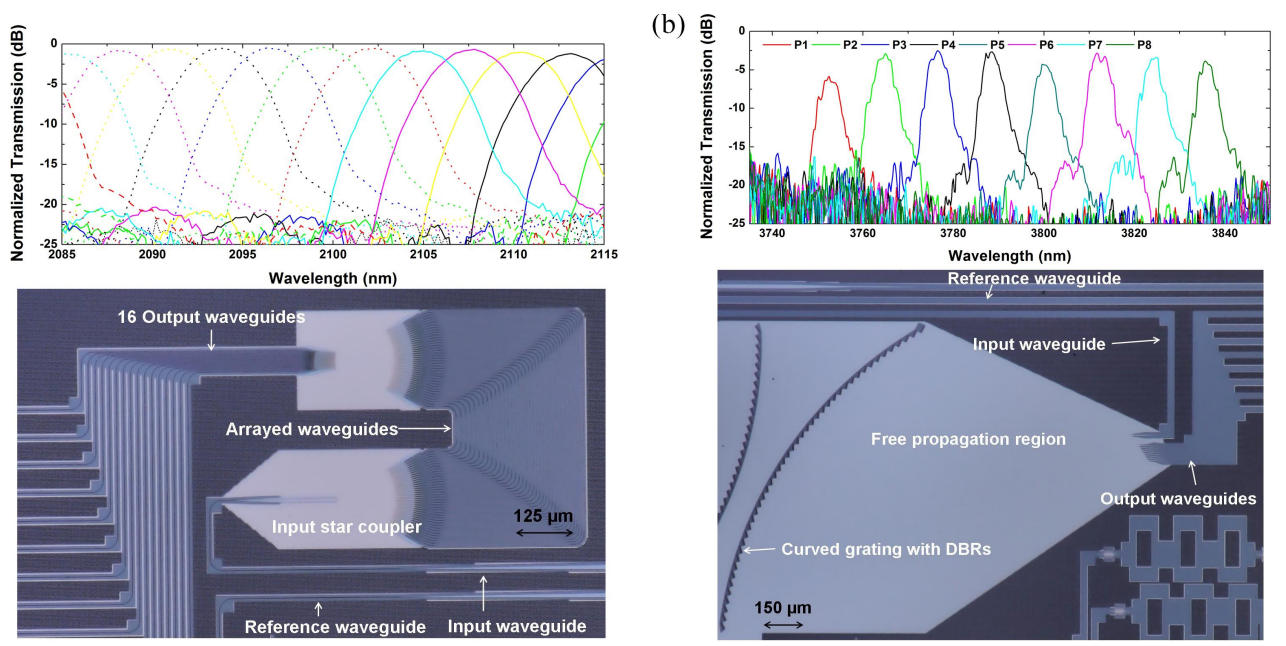

Figure 1: (a) normalized transmission spectrum and microscope picture of an integrated wavemeter operating in the 2-2.5 $\mu m$ wavelength range; (b) normalized transmission spectrum and microscope picture of a planar concave grating operating at $3.8 \mu \mathrm{m}$.

Moving beyond the $4 \mu \mathrm{m}$ wavelength range, silicon-on-insulator waveguide structures can no longer be used, due to the strong absorption of $\mathrm{SiO}_{2}$. There are different solutions to realize high index contrast waveguide structures based on silicon for this wavelength range: free-standing silicon waveguide structures [6], silicon-on-sapphire waveguide structures [7], silicon-on-nitride waveguides [8] or all-silicon waveguides [9]. The approach that we took was to use germanium-on-silicon waveguide structures for the mid-infrared [10]. Despite the large lattice mismatch between Ge and $\mathrm{Si}$, the epitaxial growth of germanium on silicon is well mastered in a CMOS fab and allows micrometer thick films with low threading dislocation density on $200 \mathrm{~mm}$ or $300 \mathrm{~mm}$ wafers. Using such a waveguide platform low-loss waveguides and integrated spectrometer circuits were demonstrated, as shown in Figure 2 [11,12].
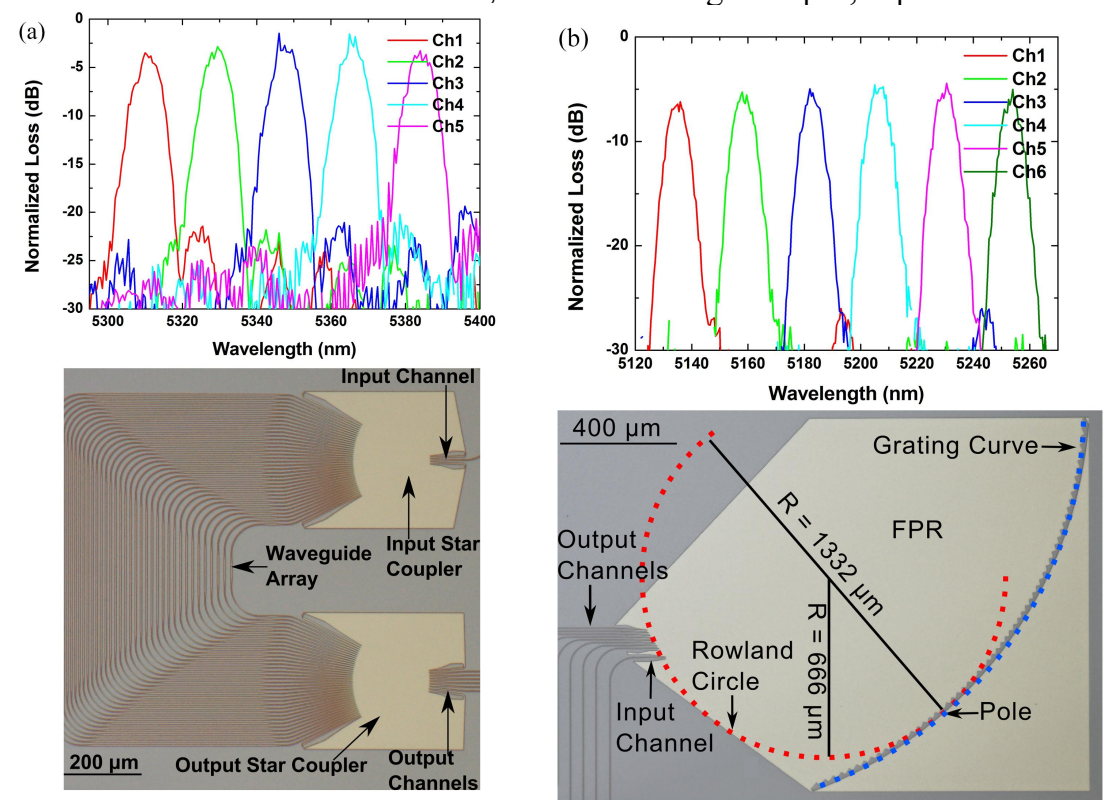

Figure 2: (a) normalized transmission spectrum of a germanium-on-silicon arrayed waveguide grating spectrometer operating in the 5-5.5 $\mu \mathrm{m}$ wavelength range and a microscope picture of the fabricated device; (b) normalized transmission spectrum of a germaniumon-silicon planar concave grating and a microscope picture of the fabricated device. 


\section{HETEROGENEOUS INTEGRATION FOR ADVANCED MID-IR PICS}

While a silicon-based waveguide platform is interesting from the point of view of manufacturability, it lacks the required integrated laser sources and photodetectors for the mid-infrared wavelength range. These components need to be added in a heterogeneous way in order to complete the toolkit for mid-infrared photonic integrated circuits. For the laser source integration we focus on the use of GaSb-based laser diodes operating in the 2-2.5 $\mu \mathrm{m}$ wavelength range. The integration is based on a III-V to silicon bonding process using DVS-BCB, a commercially available polymer, as the bonding agent. This technology was originally developed for the integration of InP-based laser sources on silicon photonic circuits [13], but was ported to the GaSb platform. The absorption of DVS-BCB is reasonably low in this wavelength range. Similar to $\mathrm{InP}$ on $\mathrm{Si}$ lasers, there is a large design freedom in the laser architecture and how to couple light between the III-V and silicon layer. In this work we focus on hybrid lasers, which require the least demanding III-V processing technology. In these devices the optical mode is mainly confined in the silicon waveguide core and the evanescent tail of the mode feels the gain in the InGaAsSb quantum wells, as shown in Figure 3(b). This first generation device lases in pulsed operation at room temperature at $2.4 \mu \mathrm{m}$ wavelength.
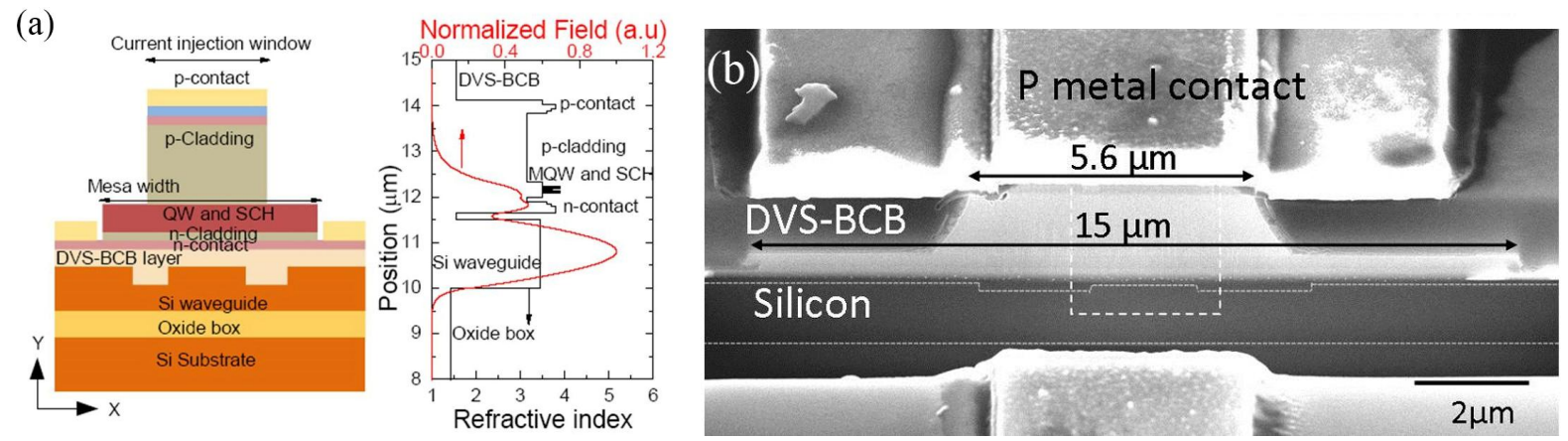

Figure 3: (a) Schematic of the hybrid GaSb on silicon laser and the device mode profile; (b) SEM cross-section of the fabricated device.

On the detector side, there are many options that can be explored, depending on the required wavelength range, required sensitivity and cost. Similar to the laser, GaSb-based photodetectors can be used for the 2-4 $\mu \mathrm{m}$ wavelength range. Using the same bonding technology as for the laser, arrays of GaSb photodetectors were integrated on silicon spectrometers as to cover $1.5-2.3 \mu \mathrm{m}$, a wavelength range that covers overtones of many important biomolecules and where water absorption is still fairly low. Figure 4 shows a microscope picture of the fabricated spectrometer chip and the performance of the integrated system $[14,15]$.
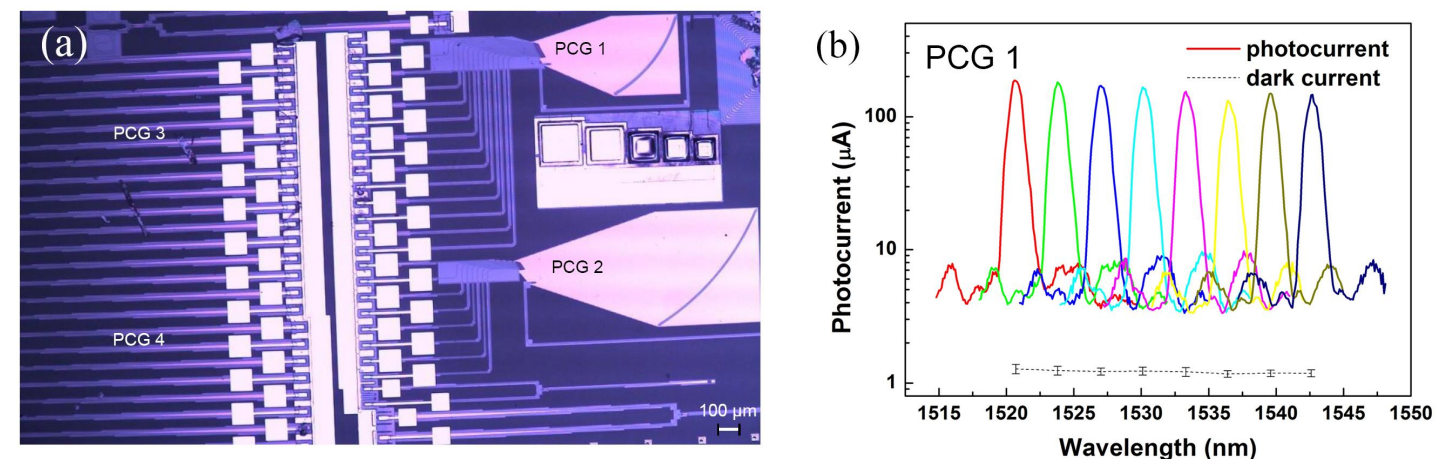

Figure 4: (a) microscope picture of the 46 channel integrated spectrometer covering the 1.5-2.3 $\mu$ mavelength range; (b) Photocurrent versus wavelength for PCG1, representative for all four spectrometers. The dark current at room temperature of the detectors is also indicated.

Next to GaSb-based devices, which are very well suited for high-sensitivity detection in the mid-infrared, alternative technologies are being explored, which aim at lower-cost (but perhaps lower-sensitivity) systems. One approach is based 
on the use of colloidal IV-VI semiconductor quantum dots such as PbS, PbSe and HgTe to realize integrated midinfrared photoconductors. These nanoparticles are synthesized in a chemical solution, thereby avoiding costly epitaxial growth of the materials. Since the dots are in solution, they can easily be applied by spincoating or dropcasting, or in a more advanced way, by using inkjet printing. The as-formed films on silicon are non-conductive, due to the long organic ligands that surround the dots (which prevents them from clustering in solution). A ligand exchange procedure was developed to replace these long organic ligands by short inorganic ones, rendering the film photoconductive. Due to the high internal gain in the photoconductor, responsivities of over $200 \mathrm{~A} / \mathrm{W}$ were measured under surface normal operation, as shown in Figure 5. Using PbS dots a cut-off wavelength up to $2.5 \mu \mathrm{m}$ has been obtained so far, while using HgTe dots we have demonstrated operation up to $3 \mu \mathrm{m}$ wavelength [16].
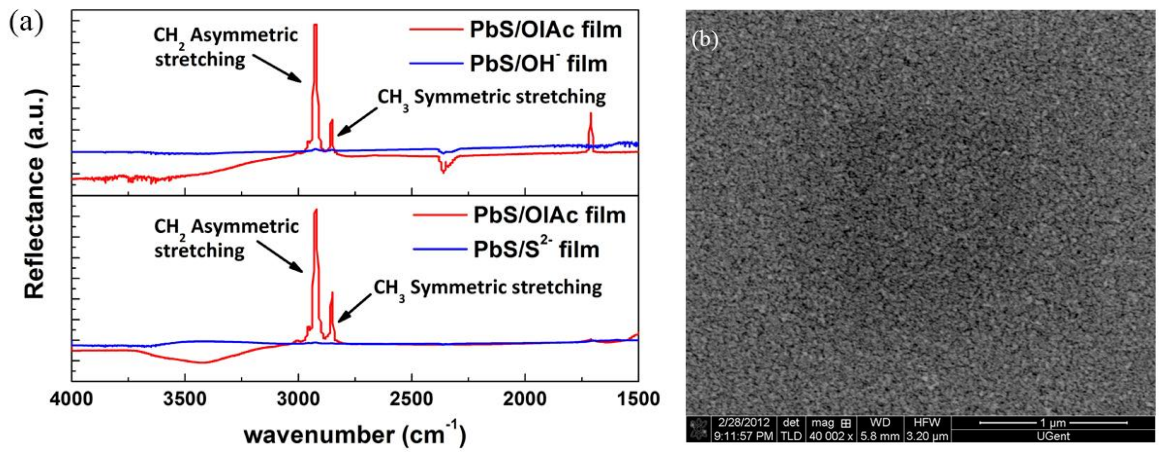

Figure 5: (a) FTIR absorption spectrum of a colloidal nanoparticle film before ligand exchange (red curves) and after ligand exchange (blue curves) - the peaks observed in the spectrum indicate absorption from $\mathrm{C}$-H bonds coming from the oleic acid (OlAc) ligands, which are absent after ligand exchange; (b) SEM picture illustrating the good surface morphology based on the developed layer deposition technique.

Eventually, a monolithic approach towards integrating a laser source and photodetector on a silicon waveguide circuit will lead to the lowest cost and the largest degree of integration. In Group IV photonics this can potentially be realized using GeSn epitaxial growth on Ge buffer layers. Adding Sn to the Ge matrix reduces the bandgap and the energy difference between the $\Gamma$ and L-valley, which are two attractive features for laser emission in the short-wave infrared [17]. As a starting point, we evaluated the performance of GeSn-based photoconductors operating in the short-wave infrared. The responsivity versus wavelength for the different device structures is shown in Figure 5(a). The absorbing region consisted of $N \mathrm{Ge}_{0.91} \mathrm{Sn}_{0.09}$ quantum wells $(N=0 . .3)$ surrounded by Ge. Clearly, adding the GeSn to the stack extends the cutoff wavelength to $2.4 \mu \mathrm{m}$, compared to the $1.7 \mu \mathrm{m}$ cut-off wavelength of Ge [18]. Since $\mathrm{Ge}$ is transparent in this wavelength range, low loss waveguides can be implemented in the Ge/GeSn on Si platform, as shown in Figure 6(b).
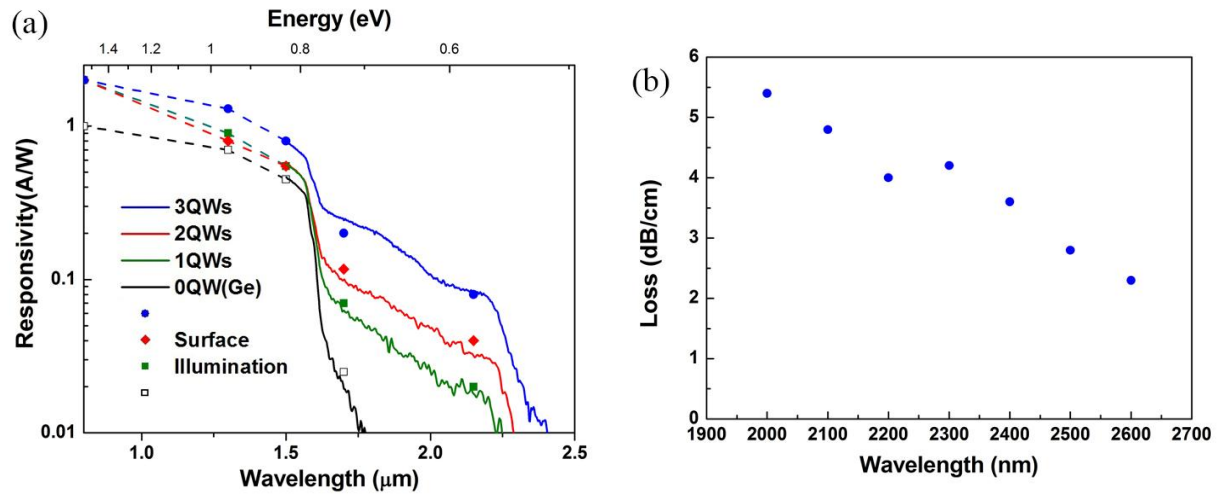

Figure 6: (a) GeSn/Ge photoconductor integrated on a $200 \mathrm{~mm}$ silicon wafer demonstrating the potential of GeSn heterostructures for the realization of short-wave infrared opto-electronic devices on silicon photonic integrated circuits: responsivity as a function of wavelength and as a function of the number of GeSn quantum wells; (b) waveguide loss of germanium-on-silicon waveguides in the 2.0 to $2.6 \mu \mathrm{m}$ wavelength range. 


\section{MID-IR NONLINEAR OPTICS}

Since silicon-based high contrast waveguide structures can tightly confine mid-infrared light and have low propagation losses, one can envision using these devices for nonlinear optical devices, especially since the Kerr nonlinearity of both $\mathrm{Si}$ and Ge is high in the mid-infrared and parasitic two-photon absorption disappears by moving to longer wavelength. This was exploited in silicon-on-insulator waveguides to obtain a 1.5-2.5 $\mu \mathrm{m}$ supercontinuum source [19] as shown in Figure 7. Besides supercontinuum sources, other devices have been realized such as a fiber-loop based silicon photonics optical parametric oscillator, which is tunable by changing the delay in the fiber loop [20]. This demonstrates that silicon nonlinear devices in the mid-infrared enable the realization of integrated light sources for mid-IR spectroscopy.

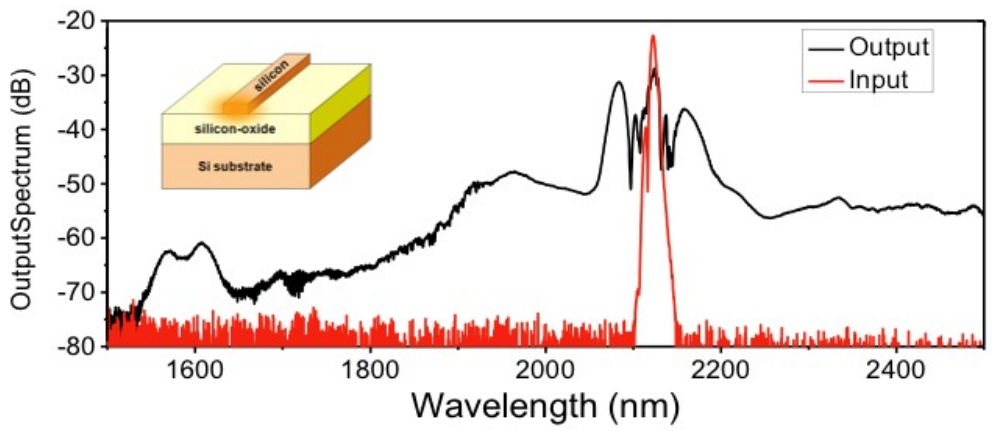

Figure 7: Supercontinuum generation in a $2 \mathrm{~cm}$ long silicon wire using picosecond pulses at $2120 \mathrm{~nm}$ with a peak power of $12.7 \mathrm{~W}$

\section{REFERENCES}

[1] C. Gunn, "CMOS photonics for high-speed interconnects", IEEE Proceedings of Computer Science, vol. 26, pp. 58 - 66, 2006.

[2] K. De Vos, J. Molera, T. Claes, Y. De Koninck, S. Popelka, E. Schacht, R. Baets, P. Bienstman, "Multiplexed antibody detection with an array of silicon-on-insulator microring resonators," IEEE Photonics Journal, 1(4), 225-235 (2009).

[3] J. G. Crowder, S. D. Smith, A. Vass, and J. Keddie, "Infrared methods for gas detection," in Mid-infrared Semiconductor Optoelectronics (Springer-Verlag, 2006).

[4] N. Hattasan, B. Kuyken, F. Leo, E. Ryckeboer, D. Vermeulen, G. Roelkens, "High-efficiency SOI fiber-to-chip grating couplers and low-loss waveguides for the short-wave infrared," IEEE Photonics Technology Letters, 24(17), 1536-1538 (2012)

[5] M. Muneeb, X. Chen, P. Verheyen, G. Lepage, S. Pathak, E. Ryckeboer, A. Malik, B. Kuyken, M. Nedeljkovic, J. Van Campenhout, G. Mashanovich, G. Roelkens, "Demonstration of silicon on insulator mid-infrared spectrometers operating at 3.8um," Opt. Express, 21(10), 11659-11669 (2013)

[6] Z. Cheng, X. Chen, C. Y. Wong, K. Xu, H. K. Tsang, "Mid-infrared suspended membrane waveguide and ring resonator on silicon-oninsulator," IEEE Photonics Journal 4(5), 1510-1519 (2012).

[7] T. Baehr-Jones, A. Spott, R. Ilic, A. Spott, B. Penkov, W. Asher, M. Hochberg, "Silicon-on-sapphire integrated waveguides for the midinfrared," Opt. Express, 18(12), 12127-12135 (2010)

[8] S. Khan, J. Chiles, J. Ma, S. Fathpour, "Silicon-on-nitride waveguides for mid- and near-infrared integrated photonics," Appl. Phys. Lett., 102, 121104 (2013).

[9] P. Lin, V. Singh, J. Hu, K. Richrdson, J. Musgraves, I. Luzinov, J. Hensley, L. Kimerling, A. Agarwal, ”Chip-scale mid-infrared chemical sensors using air-clad pedestal silicon waveguides," Lab Chip, 13, p.2162 (2013).

[10]Y. Chang, V. Paeder, L. Hvozdara, J.M. Hartmann, H. P. Herzig, "Low-loss germanium strip waveguides on silicon for the mid-infrared," Opt. Lett. 37(4), 2883-2886 (2012).

[11]A. Malik, M. Muneeb, S. Pathak, Y.Shimura, J. Van Campenhout, R.Loo, G. Roelkens, "Germanium-on-Silicon Mid-infrared Arrayed Waveguide Grating Multiplexers," IEEE Photonics Technology Letters, 25(18), p.1805-1808 (2013)

[12] A. Malik, M. Muneeb, Y.Shimura, J. Van Campenhout, R.Loo, G. Roelkens, "Germanium-on-Silicon Planar Concave Grating Wavelength (de)multiplexers in the Mid-Infrared," Applied Physics Letters 103(16), p. 161119 (2013)

[13] G. Roelkens, L. Liu, D. Liang, R. Jones, A. Fang, B. Koch, J. Bowers, III-V/silicon photonics for on-chip and inter-chip optical interconnects, Laser \& Photonics reviews (invited), p.DOI: 10.1002/lpor.200900033 (2010)

[14]E. Ryckeboer, A. Gassenq, M. Muneeb, N. Hattasan, S. Pathak, L. Cerutti, J-B Rodriguez, E. Tournie, W. Bogaerts, R. Baets, G. Roelkens, "Silicon-on-insulator spectrometers with integrated GaInAsSb photodiodes for wide-band spectroscopy from 1510 to $2300 \mathrm{~nm}$," Opt. Express, 21(5), 6101-6108 (2013)

[15]A. Gassenq, N. Hattasan, E. Ryckeboer, J.B. Rodriguez, L. Cerutti, E. Tournié, G. Roelkens, "Study of evanescently-coupled and gratingassisted GaInAsSb photodiodes integrated on a silicon photonic chip," Opt. Express, 20(11), 11665-11672 (2012)

[16]C. Hu, A. Gassenq, Y. Justo, S. Yakunin, W. Heiss, Z. Hens, G. Roelkens, "Short-wave infrared colloidal quantum dot photodetectors on silicon," 2013 Photonics West Conference, United States, p.8631-79 (2013)

[17]G. Sun, R. Soref, H. Cheng, "Design of a Si-based lattice-matched room-temperature GeSn/GeSiSn multi-quantum well mid-infrared laser diode, "Optics Express 18(10), 19957-19965 (2010)

[18]A. Gassenq, F. Gencarelli, J. Van Campenhout, Y. Shimura, R. Loo, G. Narcy, B. Vincent, G. Roelkens, "GeSn/Ge heterostructure short-wave infrared photodetectors on silicon," Opt. Express, 20(25), 27297-27303 (2012) 
[19]B. Kuyken, X. Liu, R. M. Osgood Jr., R. Baets, G. Roelkens, W. M. J. Green, "Mid-infrared to telecom-band supercontinuum generation in highly nonlinear silicon-on-insulator wire waveguides," Opt. Express, 19(21), 20172-20181 (2011)

[20] B. Kuyken, X. Liu, R. M. Osgood Jr., R. Baets, G. Roelkens, W. M. J. Green,”A silicon-based widely tunable short-wave infrared optical parametric oscillator," Opt. Express, 21(5), 5931-5940 (2013) 